\title{
Confocal Raman Microscopy
}

\author{
J. Müller, W. Ibach, K. Weishaupt, and O. Hollricher \\ WITec GmbH, Hoervelsinger Weg 6, 89081 Ulm, Germany
}

Raman spectroscopy is a very powerful technique that delivers detailed chemical information of the species involved in the scattering process. However, in most spectroscopy setups spacial resolution is very poor, because the exciting laser spot diameter is in the order of $100 \mu \mathrm{m}$.

Optical microscopy on the other hand is capable of providing a spacial resolution down to $200 \mathrm{~nm}$ using visible light excitation. In a confocal microscope, the light is detected through a pinhole, giving rise to depth resolution and a strongly reduced background signal.

The CRM200 combines a sensitive Raman spectrometer and a confocal microscope with extremely high optical throughput and resolution. With this instrument it is not only possible to obtain Raman spectra from extremely small sample volumes (down to $0.02 \mu \mathrm{m}^{3}$ ), but also to collect high resolution Raman images.

This is done by focusing a laser spot to a diffraction limited spot size (down to 200nm@488nm excitation), while the sample is scanned with a piezoelectric scan table. The scan table is extremely linear and precise, due to capacitive feedback linearization. The Raman scattered light is detected through an optical fiber which acts as a real confocal pinhole and is directed to a spectrometer with CCD camera and ultra-sensitive photon counting detector.

The user has the choice of Raman Fast Imaging, which is the collection of an image in the light of a single Raman line and Raman Spectral Imaging. In Raman Fast Imaging, the spectrometer is used as a spectrograph and the light of a selected Raman line is directed to the photon counting detector. While scanning the sample, the image is obtained in the light of a single Raman line.

In Raman Spectral Imaging, which the most versatile mode of the CRM200, the spectrometer is tuned to an area of interest and a CCD camera is used to aquire a complete spectrum at every image point. The number of image points (spectra) can be up to $256 \times 256=65536$ or even more, only limited by the memory of the computer.

Due to the extremely sensitive setup, the acquisition time per spectrum is typically $100 \mathrm{~ms}$ or even less, so that images of $100 \times 100$ pixels are obtained in 15 minutes.

To demonstrate the capabilities of the system, several samples from different application fields will be discussed. As an example, a CVD diamond film on silicon was investigated.

Usually, the width of the Raman line at $1333 / \mathrm{cm}$ is used to characterize the quality of the diamond film. But if the laser spot has a diameter of e.g. $100 \mu \mathrm{m}$, the width of the measured Raman line is an average over the laser spot diameter.

To investigate the sample in more detail, a $50 \mu \mathrm{m} \times 50 \mu \mathrm{m}$ area was analyzed by taking $256 \times 256=$ 65536 spectra with an integration time of 50ms. For excitation and detection we used a 100x, $\mathrm{NA}=0,9$ objective and about $10 \mathrm{~mW}$ power of a frequency doubled Nd:YAG laser at $532 \mathrm{~nm}$. The spectra were obtained with a $300 \mathrm{~mm}$ imaging spectrometer equipped with a $1800 \mathrm{lines} / \mathrm{mm}$ grating and a back-illuminated CCD camera. The complete image aquisition took less than one hour.

Fig. 1 shows the fluorescence due to impurities which mainly occures at the grain boundaries. The image was calculated by integrating over the complete intensity in each spectrum. Fig. 2 shows the Raman intensity in the diamond line obtained by integrating over the diamond line in each spectrum, corrected for the fluorescence background. As one can see, the Raman intensity (sum of the Raman scattered signal) is relatively homogenous, and not all grain boundaries show a strong fluorescence. 
However, the width of the Raman line shows strong local variations as shown in Fig.3. The bright areas correspond to areas with small line widths, while the dark areas show broadened Raman lines. The FWHM varies from about $5 / \mathrm{cm}$ (resolution due to entrance slit of the spectrometer) to more than $60 / \mathrm{cm}$.

Fig. 4 shows different spectra from points $\mathrm{A}, \mathrm{B}$ and $\mathrm{C}$ respectively as indicated in Fig. 3. Spectra from the bright areas in Fig. 3 (A) are located at 1333/cm and have a very small line width as expected for pure diamond. Spectra from the dark areas (B and C) show a strong broardening and a splitting into two (B) or even 3 lines (C). For comparison, the spectrum averaged over all 65536 spectra is shown. This spectrum would have been obtained, if a laser spot diameter of $50 \mu \mathrm{m}$ would have been used. As one can see, the averaged spectrum shows a FWHM of $14,6 / \mathrm{cm}$, which is a significant broardening to the spectrum from (A) which shows a line width of only $6,5 / \mathrm{cm}$.

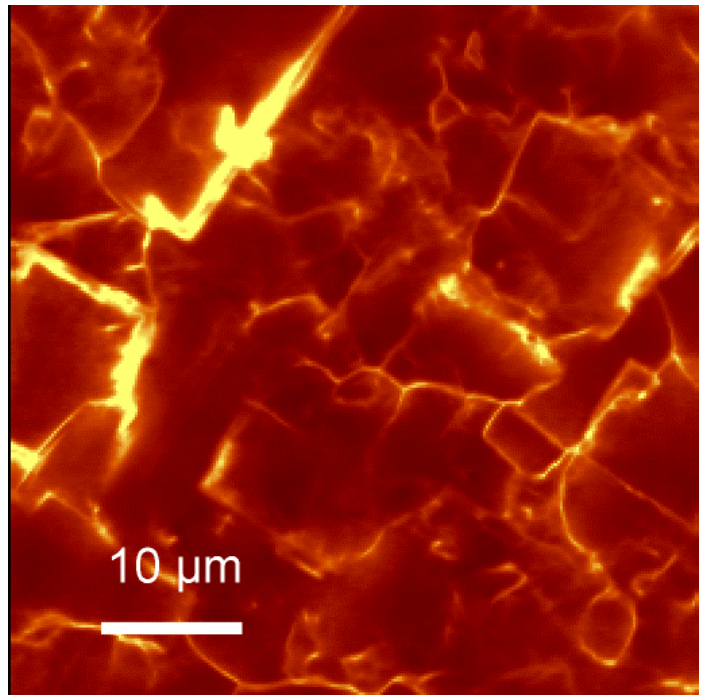

Fig. 1: Fluorescence intensity

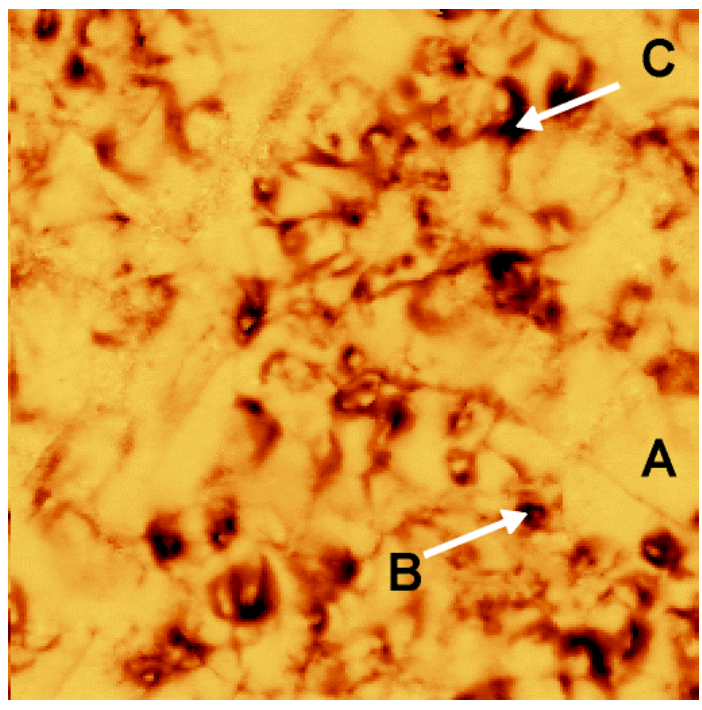

Fig. 3: FWHM of the diamond line (dark=high)

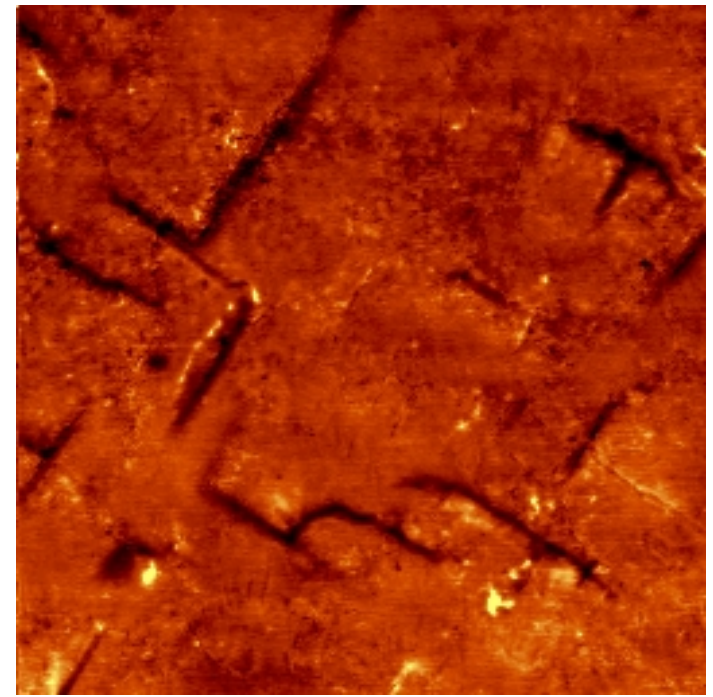

Fig. 2: Raman intensity of the diamond line

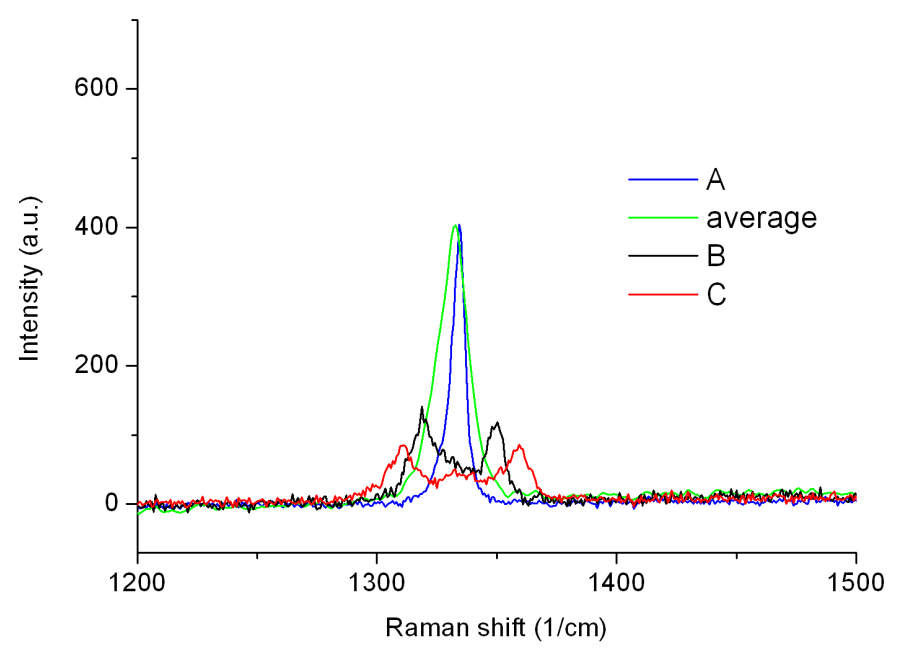

Fig. 4: Raman spectra at different points 